THEORIA ET HISTORIA SCIENTIARUM, VOL. VIII, N" 2

Ed. Nicolaus Copernicus University 2008

Phillip Okwo

\title{
How the Effect of Emotion on the Selectivity of Attention Supports Self-Organizing Dynamical Systems as an Explanation of Consciousness
}

\begin{abstract}
This paper attempts to provide a systematic argument, on both philosophical and neuropsychological grounds, for an "enactive" and self-organizational explanation for consciousness. The self-organizational approach offers solutions to problems that have stumped philosophers for centuries, including the problem of mental causation. Mental causation is a mystery because it seems that the unified being, the conscious person or animal, is able to make its own parts combine in certain ways to serve the purposes of the whole, rather than having its form merely dictated by the accidental ways in which the constituents come together. The theory of self-organization explicitly addresses this problem as its basic starting point. Also, many psychological puzzles about consciousness can be addressed by means of a self-organizational approach. In this paper, we consider the Mack and Rock inattentional blindness phenomena; and the micro-genesis of perceptual experience, which presents the paradox that perceptual consciousness does not accompany virtually complete occipital activation, yet does accompany the parietal P300 event related potential, which however does not seem to be caused by the occipital activation at all. We also explore the way the enactive, dynamical systems account of emotional consciousness maps onto the relatively independent, unconditioned instinctual systems studied by Panksepp and associates, as discussed in the target article of this issue.
\end{abstract}

Key Words: Dynamical systems; emotion; consciousness; mental causation; micro-genesis; inattentional blindness; event related potentials; attention; enactive theory.

Self-organizing dynamical systems, simply stated, are systems that "seek out, appropriate, and replace its own physical substrata via an inherent, self-organizing 
tendency to actively rearrange the background conditions that make different substrata possible as necessary in order to ensure the continuity and structural pattern of the entire system" (Ellis, 2001a). Such systems are autonomous with respect to their components and can be described as "top-down" in their constitution. Self-organizing dynamical systems, in other words then, are systems that exert some control over the parts that compose them in order ultimately to maintain themselves.

An example of a self-organizing dynamical system that helps to better demonstrate what self-organizing dynamical systems are and how they differ from other types of systems is the maintenance of a living organism's constant temperature (Ellis, 2001b). The maintenance of constant temperature is selforganizing, because when the temperature gets too hot or too cold, the organism must systematically readjust many other activities throughout the system in order to maintain the temperature. Shivering when one is cold is one of the body's such readjustments of activities that actually helps to generate heat. If an organism does not readjust its activities to maintain temperature, the survival of the definitive structure of the system as a whole is threatened. In the case of thermostats, on the other hand, though they maintain constant temperature, it is not necessary for them to be self-organizing to produce the results that they do, because the action of each part does not depend on the whole readjusting some parts to compensate for the actions of others.

Another example of a self-organizing dynamical system is that of biochemical pathways. Biochemical pathways are successful on the basis of their ability to use multiple combinations of different substrates in order to reach the ultimately desired end product.

But what do self-organizing dynamical systems have to do with consciousness? The nature of human consciousness has been one of philosophy's roaring debates for centuries now. Many explanations and theories have been offered, and so far none of them can really be deemed adequate. This is where self-organizing dynamical systems come in. Self-organization has been emerging as an explanation, and there are indications that self-organizing dynamical systems are an ideal explanation for the nature of human consciousness.

In addition to the fact that past theories that have been offered so far have involved internal logical problems - such as the famous problems of mental causation, the apparent sufficiency of physical explanations which renders mental explanations redundant, and the public inaccessibility of subjective consciousness in spite of the public nature of its supposed physical equivalents - selforganization seems promising in its ability to answer the questions and tackle the problems that the other theories have either raised themselves or have thus far proven themselves unable to handle (Ellis, 1999). These include Chalmers's notorious "hard problem": even if we could cite necessary and sufficient causal 
antecedents for the physical correlates of a state of consciousness, we still would not explain why the physical correlates should be accompanied by consciousness rather than merely non-conscious physical events. Moreover, scientific evidence on the manner in which emotion seems to affect the selectivity of attention seems to lend still further credence to the possibility that self-organizing dynamical systems are in fact the way to go in seeking to explain the nature of human consciousness. The ways that emotion has been demonstrated to affect the selectivity of attention will be the focus of the next part of this paper.

More and more recent scientific evidence is indicating that emotion (and motivation) affects the selectivity of our attention, and to a significant degree. I think it is reasonable to assume that a careful examination of such evidence and its implications will shed much light on the way our consciousness might be explained.

One such study that looked into the selectivity of our attention is the inattentional blindness study performed by Mack and Rock. "In a typical experimental design, the research subjects are asked to determine which arm of a cross is longer than the other, and then while they are involved in this task a small image is presented in various places within or near this zone of attention, for a short time in the neighborhood of 200 milliseconds. In many of the experimental paradigms, most subjects fail to see the extraneous image, even though control subjects (whose attention is not otherwise occupied) consistently see it quite clearly. When asked to pick out the critical stimulus from an array of alternatives, their performance did not differ from chance" (Mack \& Rock, 1998). I.e., subjects exhibiting inattentional blindness in most instances "did not even show implicit knowledge of the unseen stimulus, at least as far as Mack and Rock could detect. Again this does not necessarily mean that there is no implicit perception, but the explicit failure to detect it must be taken into account" (Ellis, 2001b).

This failure to see objects within the visual field when attention is otherwise occupied suggests that there is no conscious perception without attention. The findings of research performed by Aureli support the findings of Mack and Rock. This research shows that "following the presentation of a novel stimulus... there can be virtually complete occipital processing, but until and unless what is called a P300 parietal eventrelated potential occurs in the parietal lobe, the subject is not conscious of seeing the presented stimulus" (Aureli, 1989). In other words, a separate attentional act is required before any visual information can be registered in consciousness, even after it his been fully processed in the perceptual brain areas.

These findings in combination with others have proven to be consistent with what Varela et al. have called an "enactive" theory of consciousness, in which, as Ellis elaborates the theory, "emotional activation of subcortical and anterior 
brain areas is necessary before conscious information processing can occur" (Ellis, 1999). Furthermore, an enactive theory that explains this level of phenomenon entails that an organism that behaves as such must be an active, self-organizing entity. In this instance, the self-organizing system "initiates actions geared toward using the environment as needed to keep the system going in its definitive pattern" (Ellis, 2001b) - for example, determining which arm of a cross is longer.

What is important to note, and was definitely demonstrated by Mack and Rock, is the fact that when our attention is consciously directed toward one task, although irrelevant stimuli had a tendency not to be readily processed, this was not the case with: (1) emotionally salient stimuli (for example, a smiling face - which subjects did respond to while attending to the cross); (2) meaningful stimuli (for example, the written words that yield implicit perception; may be a meaningful "icon," at least in Western cultures); or (3) types of objects that are rigidly hardwired for selection in terms of very gross properties, such as loud noises, very large moving objects, sudden bright lights, etc. (Mack \& Rock, 1998).

The first two of these exceptions may result as a consequence of a process called "valence-tagging" which will be discussed at greater length later in this paper. All three types of exceptions, though, remain compatible with the idea of self-organizing dynamical systems, as we shall soon see.

Even before Mack and Rock conducted their experiments, de Souza hypothesized that "the emotions function to direct our attention" (de Souza, 1987). Faucher and Tappolel examined this hypothesis and its implications in the wake of research conducted specifically on the effects that emotions, such as fear and longer-term moods or states (such as anxiety disorders and phobias), may have on what information is processed by an individual. Relevant research included homophone spelling tasks, emotional Stroop tasks, dot probe detection tasks, and pop-out tasks.

Homophone spelling tasks, as conducted by Mathews, et al., consist of "writing down single words that they heard [where] some of the words were homophones: one of its meanings was threat-related ("die"), whereas the other was neutral ("dye")" (Mathews \& Eysenck, 1989). Subjects with trait anxiety and particular anxiety disorders demonstrated an increased attentional bias towards threat-related stimuli by writing down more threat-related homophones than did controls.

In the emotional Stroop tasks of Mogg et al. subjects were shown words printed in different colors. They were asked to ignore the word content and to name the color as quickly as possible. Results reported that subjects with various forms of anxiety took longer than controls to recognize the color of words. Furthermore, trait anxiety and generalized anxiety disorder subjects took even "longer to name the colors of threatening words, such as 'cancer' or 'collapse."' (Mogg, Mathews, \& Weinmann, 1989). Particular anxiety disorders exhibited 
interference in recognizing the color of words that were related to the objects of their anxiety such that arachnophobic subjects had trouble identifying the colors of spiderrelated words. This evidence seemed to indicate that more attention is given to the content of these threat-related words.

MacLeod et al. (1986) developed dot probe detection tasks involving the presentation of word pairs on a screen - with one word appearing in the upper part of the screen and the other word appearing in the lower part of the screen - for a time of half of a second. Sometimes the presented word pairs consisted in one threat-related word and the other a neutral word. After the half-second delay, a dot probe replaced one of the words. Subjects were asked to indicate by pressing a button as soon as they detected the dot probe. Because subjects tended to respond faster to probes appearing in the region of their attention, the evidence that subjects with trait and particular anxiety conditions responded faster when the dot probe appeared in the area where threat-related words were presented (MacLeod, Mathews, \& Tata, 1986) is not surprising. Additionally, an interesting finding was that these same subjects responded faster when the dot probe appeared near positive words when juxtaposed with neutral words than did control subjects.

Finally, Ohman and colleagues used pop out tasks to investigate the effect of emotion on attention. In these tasks, subjects were showed images made of nine pictures distributed into three rows of three. Eight of these pictures would be similar and one would be an intruder. These researchers found that "reaction times were shorter for a frightening intruder than for a neutral stimulus" (Ohman, 2000) in the case of all subjects. This attentional bias was magnified when subjects with particular anxiety disorders or phobias were presented with an intruder that was related to the object of their anxious or fearful disposition. In these instances, subjects responded even faster than when the intruder was threat-related, though not related to their phobia or particular anxiety disorder.

Faucher and Tappolet's juxtaposition and examination of this research reveals a good deal of information about the manner and degree in which the powerful emotions of fear and anxiety influence attention, thereby bringing the object that is to be feared into the consciousness of the individual. "Part of a normal fear response serves to attract attention to environmental threat cues, thus facilitating the acquisition of threatening information but also consists in orienting towards relieving cues - such as finding an escape route, a stick, a place to hide - as was demonstrated in the subjects of the dot probe detection tasks" (Faucher \& Tappolet, 2002). In addition, it is of note that generalized anxiety disorder, trait anxiety, and particular anxiety disorders are associated with attentional biases towards congruent stimuli (Faucher \& Tappolet, 2002) reminiscent of how a selforganizing dynamical system, again, initiates actions geared toward using the environment as needed to keep the system going in its definitive pattern (in this case, an attentional bias towards spiders, snakes, etc. contributes to the 
maintenance of a pattern of mental functioning that includes anxiety or fear of spiders, snakes, or everything in general, respectively).

As has been shown, a significant amount of research evidence on the manner in which emotion affects the selectivity of attention contributes to this idea that consciousness could feasibly be explained as a self-organizing dynamical system. However, the consideration of much of the research that suggests interplay between emotions and consciousness in other, sometimes more broader, aspects is not taken as seriously as the preponderance of such findings would suggest they should. Indeed, if more of the cognitive neuroscience research were considered in accord with its prevalence, as it should be, not only would it probably reveal even more ways in which consciousness displays attributes of a self-organizing dynamical system, but it would also probably demand a much more comprehensive approach to cognitive neuroscience research than is currently being taken. Some important research findings that deserve greater consideration and their implications will be discussed below.

In a paper called "The Neuro-Evolutionary Cusp Between Emotions and Cognitions," Jaak Panksepp argues - on the basis of research findings that he feels to be commonly overlooked - for a unified mind science, which would bring greater focus to the subcortex's relationship with emotions and motivation and the manner in which these affect cognition, in order to allow the whole brain and not only the cortex to truly have a role in the pursuit of substantive knowledge in the area of consciousness.

Having examined the common behavior in all mammals as demonstrating some degree of subjectivity and of "avidly consuming and getting 'hooked' on the same drugs that promote similar neuropsychological processes as in humans" (Panksepp, 2000), Panksepp is convinced that subcortical substrates, which are the source of basic emotions, are essential for these desires. These findings, he maintains, make it reasonable to believe that these behaviors and basic emotions reflect organizational principles of "neural systems whose substrates constitute the very foundation of all subsequent forms of consciousness." Panksepp rejects the cortico-centric modern cognitive neuroscience in as much as it ignores this evidence and its implications.

Panksepp explores valence-tagging, or the attribution of values to initially emotionally neutral events, which normally occurs from classical conditioning, as "one of the simplest forms of cognitive-emotional interaction that exists within the brain" (Panksepp, 2000). Via this interaction, it is possible for an individual to learn on a cognitive level "what they like and what they do not like" when they previously only know on an instinctual level.

Moreover, Panksepp's exploration of projection - the tendency of people to cast their feelings onto the world - ultimately leads to speculation on how consciousness could be an evolutionary possibility. Projection, he theorizes, may 
be a "pervasive and dynamic brain response, reflecting direct actions of emotions on brain areas that mediate cognitive and perceptual processes. And, since cognitive processes are designed to deal with moment by moment events in the external world, while affective feelings reflect evolutionarily provided value codes, it may be that the projection of feelings onto environmental events and objects was one of the simplest ways for evolution to persistently guide the priorities of the cognitive apparatus" (Panksepp, 2000).

Indeed, Panksepp insists that "cognitive forms of consciousness were evolutionarily premised on the prior evolution of affective forms of consciousness which would inform us what it might be worth thinking about" (Panksepp, 2000).

More importantly, Panksepp demonstrates that an evolutionary explanation for the origins of consciousness does not necessarily preclude the possibility of free will. As the processes of affective consciousness required at least the brain structures that are present in all mammals, additional layers of brain evolution were required to create even higher levels of cognitive processes. Because free will entails the "capacity to entertain several conflicting emotional and motivational alternatives concurrently and facilitate adaptive response selection from alternative courses of action, it is clear that higher brain systems are able to deliberate more fully on affective issues than they could without the higher symbolic capacities of the cortico-cognitive apparatus" (Panksepp, 2000). Free will, then, exists because a sufficient degree of higher-order cognitive process has developed from the foundation of the subcortically situated core mechanism for affect, and it can be readily seen how evolution would select for free will.

Interestingly, taking into account these possibilities of the evolution of consciousness and free will, one could ask whether it is taking too much liberty to speculate that consciousness might be a simple extension of the manner in which human beings as organisms are self-organizing dynamical systems similar to other selforganizing dynamical features such as thermostatic regulation and the mechanisms of biochemical pathways.

And, finally, as Panksepp suggests, by giving these research findings their due consideration, one can form a better idea of how consciousness might exist, particularly as a self-organizing dynamical system. Panksepp's synopsis of affective neuroscience - which proposes that subcortical neural organization generates the basic desires and has a pervasive influence on consciousness; that higher cortical processes do, also, affect the emotional subcortical processes mainly in a regulatory or modulatory capacity; that cognitive forms of consciousness are evolutionarily premised on prior evolution of affective forms; and that centromedial mesencephalic systems of the brain (e.g. the periaqueductal gray, or anterior cingulate) are the most likely areas of the brain to explain our affective experience or consciousness of our basic emotions, due to their location at an area where the cortical and subcortical areas of the brain converge, and 
consequently their potential capacity to serve as a relay of information from one of these areas to the other and vice versa. These principles of brain organization, with the emotional areas driving the process for purposes of holistic, self- organizational balance of the system, help to demonstrate the attributes that systems in which emotions affect cognitive processes in such various ways would be expected to possess: "they are able to (1) directly evaluate the meaning of sensory inputs (e.g., the smell of predators in prey species); (2) they modulate attentional and sensory-motor sensitivities relevant for the evoked behavioral tendencies (e.g., hunger sensitizes olfactory acuity); (3) they control diverse physiological and hormonal conditions of the body which bring many organ systems in line with the concurrent behavioral demands (e.g., adrenaline secretion is adaptive for all behaviors that require motor arousal); (4) they sustain animals in specific feeling (mood) states for relatively prolonged periods of time (e.g., separation protest vocalizations and feelings of distress are typically sustained until social reunion occurs or despair sets in). All of the above are also (5) modulated by various cognitive activities (i.e., appraisals can provoke emotions) and which (6) also modulate cognitive activities, and, finally, (7) the existence of affective experience" (Panksepp, 2000).

In light of this synopsis, in addition to Panksepp's research and that of others presented above, it becomes increasingly easy to formulate possibilities of mechanisms or frameworks in which the brain might be structured to produce consciousness in line with the self-organizing dynamical explanation.

The demonstrated seriousness with which self-organizing dynamical systems should be considered as an explanation of consciousness has several implications both philosophical and neurophysiological.

The neurophysiological implications are that the current focus of research is particularly short-sighted, and research will have to be given greater, more comprehensive consideration even in the face of societal pressures on research if any meaningful and substantive progress beyond this point is to be made in the field with regard to a proper understanding of consciousness.

Philosophical implications, aside from the fact that prior philosophical theories in the philosophy of mind might reasonably be abandoned, include the possibility that the Cartesian dualist metaphysical explanation of the nature of the reality would now lose whatever explanatory attraction it might once have possessed. Many philosophers were reluctant to fully embrace physicalist explanations because they could not address Charlmers's "hard problem," the "explanatory gap," the "knowledge argument," etc. But self-organizing dynamical systems make possible an explanation of consciousness that does not end up falling into the usual trap of the mind-body problem and how mental processes can possibly effect physical actions (how do I move my arm at the thought of moving my arm?) and all of the nasty, sticky little hang-ups that lie 
therein. From this one might sensibly propose a more monist perspective on metaphysics.

A major objection to the theory that self-organizing dynamical systems might be a fitting explanation for the nature of consciousness is the metaphysical consideration that the components of these supposed systems are the only thing that can be considered real, and that the self-maintaining system itself is simply an epiphenomenon or coincidently occurring phenomenon.

This objection has been responded to by Ellis, who points out that "to declare that the self-maintaining system is 'merely an epiphenomenon' is not to say that the selfmaintaining system as just defined does not exist, nor that it does not have the tendencies described, which give it the power to maintain itself across replacements of its substrata, thus allowing a meaningful distinction between the system and its components. What the objection really entails, if its premise is true, is that self-maintaining systems have their own causal antecedents, just like everything else in nature. But to say that they are caused to be the way they are does not negate the possibility that they in turn can play a role in bringing about subsequent effects" (Ellis, 2001a).

In conclusion, self-organizing dynamical systems serve as a new hope for the possible explanation to the age-old philosophical question of the nature of consciousness. So far, the theory seems to have demonstrated solidity enough to be worth exploring seriously. As long as the research suggests this, proper scientific and philosophical endeavor demands that our efforts be applied appropriately if we expect ultimately to possess a working foundation of knowledge about consciousness that opens up the possibility of engaging consciousness to the limits of its rich capabilities, yet in a way that is also congruent with a scientific worldview.

\section{References}

Aureli, C.G., 1989: Man's triune conscious mind, Perceptual and Motor Skills 68: 747- -754

De Souza, R., 1987: The Rationality of Emotion, Bradford Books, Cambridge, Massachusetts

Ellis, R.D., 1999: Dynamical systems as an approach to consciousness: Emotion, selforganization, and the mind-body problem, New Ideas in Psychology 17: 237-250

Ellis, R.D., 2001a: Can dynamical systems explain mental causation? Journal of Mind an Behavior 22: 311-334

Ellis, R.D., 2001b: Implications of inattentional blindness for "enactive" theories of consciousness, Brain and Mind 2: 297-322

Faucher, L. and Tappolet, C., 2002: Fear and the focus of attention, Consciousness \& Emotion, 3(2): 105-144 
Mack, A. and Rock, L, 1998: Inattentioiial Blindness, MIT/Bradford, Cambridge, Massachusetts

MacLeod, C., Mathews, A., Tata, P., 1986: Attentional bias in emotional disorders, Journal of Abnormal Psychology 95: 15-20

Mathews, A., Richards, A., Eysenck, M.W., 1989: The interpretation of homophones related to threat in anxiety state, Journal of Abnormal Psychology 98: 31-34

Mogg, K., Mathews, A., Weinmann, J., 1989: Selective processing of threat cues in anxiety states, Behaviour Research and Therapy 34: 17-36

Ohman, A., 2000: La biologie des passions, Pour la science 271: 50-57

Panksepp, J., 2000: The neuro-evolutionary cusp between emotions and cognitions, Consciousness \& Emotion 1(1): 15-54 